\title{
Singular values and non-repelling cycles for entire transcendental maps
}

\author{
Anna Miriam Benini *and Núria Fagella ${ }^{\dagger}$ \\ Dept. de Matemàtica y Informàtica, Universitat de Barcelona \\ Barcelona Graduate School of Mathematics (BGSMath) \\ Gran Via 585 08007, Barcelona
}

March 25, 2022

\begin{abstract}
Let $f$ be a map with bounded set of singular values for which periodic dynamic rays exist and land. We prove that each non-repelling cycle is associated to a singular orbit which cannot accumulate on any other non-repelling cycle. When $f$ has finitely many singular values this implies a refinement of the Fatou-Shishikura inequality. Our approach is combinatorial in the spirit of the approach used by [Kiw00, $\mathrm{BCL}^{+16}$, for polynomials. प
\end{abstract}

\section{Introduction}

Consider the iteration of an entire transcendental map $f: \mathbb{C} \rightarrow \mathbb{C}$. The map $f$ fails to be a covering due to the presence of singular values, that is the set $S(f)$ of points near which not all inverse branches of $f^{-1}$ are well defined and univalent. While the singular values of rational maps are always critical values (images of zeros of $f^{\prime}$ or critical points), transcendental functions may have also asymptotic values, and we have that

$$
S(f)=\overline{\{\text { critical and asymptotic values for } f\}} .
$$

Recall that $s \in \mathbb{C}$ is an asymptotic value if there exists a curve $\gamma:[0, \infty) \rightarrow \mathbb{C}$ such that $|\gamma(t)| \rightarrow \infty$ as $t \rightarrow \infty$ and $f(\gamma(t)) \rightarrow s$ as $t \rightarrow \infty$ (for example, $s=0$ is an asymptotic value for the map $z \mapsto \exp (z)$, and the curve $\gamma$ can be taken to be the negative real axis).

Special classes of maps are singled out in terms of their set of singular values and will be important for our discusion. More precisely define

$$
\mathcal{S}=\{f: \mathbb{C} \rightarrow \mathbb{C} \text { entire } \mid \# S(f)<\infty\}
$$

\footnotetext{
*Partially supported by the Marie Curie IEF grant H2020 703269 COTRADY.

${ }^{\dagger}$ Partially supported by the Spanish grant MTM2014-52209-C2-2-P and the Maria de Maeztu Excellence Grant MDM-2014-0445 of the BGSMath.

2010 Mathematics Subject Classification. Primary 30D05, 37F10, 30D30.
} 
and

$$
\mathcal{B}=\{f: \mathbb{C} \rightarrow \mathbb{C} \text { entire } \mid S(f) \text { is bounded }\},
$$

Class $\mathcal{S}$ and class $\mathcal{B}$ are known as the Speiser class and the Eremenko Lyubich class respectively. The elements in $\mathcal{S}$ are called finite type maps while those in $\mathcal{B}$ are of bounded type.

Singular values play a crucial role to understand the dynamics of $f$. Indeed, using local dynamics it is possible to investigate the relation between singular orbits and non-repelling cycles. For example it is well known that each cycle of parabolic and attracting basins contains a singular value [Fat20, Mil06. Using normal families arguments one can also see that Cremer points and all points in the boundary of Siegel disks are contained in the accumulation set of the orbits of the singular values [Fat20, Mil06. While the orbit of a singular value which belongs to an attracting or parabolic basin is fully contained in the basin and only accumulates on the attracting or parabolic point associated to the basin, it is not clear using only local theory that a unique singular orbit cannot accumulate, for example, on many Cremer cycles or cycles of boundaries of Siegel disks.

Nevertheless, using perturbation arguments in the finite dimensional space of rational maps of degree $d \geq 2$, Fatou [Fat20] was able to show that the number of non-repelling cycles of a rational map of degree $d \geq 2$ is bounded by $4 d-2$, that is twice the number of its critical points counted with multiplicity. Afterwards he conjectured that the optimal bound should be $2 d-1$. With this goal in mind, Shishikura [Shi87] used quasiconformal surgery to perturb simultaneously all indifferent cycles to attracting ones, proving Fatou's conjecture, known nowadays as the Fatou-Shishikura inequality. A simpler proof for polynomials using perturbation in the class of weakly polynomial-like maps can be found in [DH85, while a different approach using quadratic differentials is in Eps99]. Later on, it was proven in [EL92, Theorem 5] and [GK86] that for an entire transcendental map of finite type, the number of non-repelling cycles is also bounded by the number of singular values of the map, proving the Fatou-Shishikura inequality for this class $\mathcal{S}$ of functions.

All these results, like those on the non-existence of wandering domains for these finitedimensional families, are based on arguments in parameter space, and, despite giving a sharp bound, they do not provide dynamical information on how exactly the orbits of the singular values relate to the non -repelling cycles. For example, a priori if the function has $q$ singular values and $q$ Cremer cycles there could be a unique singular value whose orbit accumulates on the $q$ Cremer cycles while the remaining $q-1$ singular values do not accumulate on any Cremer point.

A different combinatorial approach for polynomials with connected Julia sets was suggested by Kiwi Kiw00. Kiwi's approach is dynamical and associates to each non-repelling cycle a specific singular orbit. Observe that for polynomials the Julia set fails to be connected only when singular values are escaping. This is a well understood case and Kiwi's approach can be extended to the case in which the Julia set is not connected (compare with $\left[\mathrm{BCL}^{+} 16\right]$ ). Very recently another dynamical approach involving laminations and fibers was used in $\left[\mathrm{BCL}^{+} 16\right]$ to prove a more general version of the Fatou-Shishikura inequality for polynomials, which takes into account also wandering branch continua. These new approaches prove among others the following statement, which is slightly stronger than the classical Fatou-Shishikura inequality. 
Proposition $\left.1.1\left(\overline{\mathrm{BCL}^{+} 16}\right]\right)$. Let $P$ be a polynomial. Then any non-repelling cycle is associated to a weakly recurrent critical point, and distinct non-repelling cycles are associated to distinct critical points.

It is a natural question whether these stronger versions for polynomials hold also for their transcendental analogues, that is for entire transcendental maps of finite type. And even further, whether or how far they can be pushed when dealing with maps with an infinite number of singular values. This approach is inlaid in the frame of understanding whether these relationships between singular values and non-repelling cycles are intrinsically of local nature or instead rely on the global structure of the families of maps under consideration.

In this paper we combine the main results in [BF15] with classical normal families arguments and some combinatorics to give some answers to both of these questions. Our main result (Theorem 1.3) applies to maps in class $\mathcal{B}$ with some additional conditions and, when applied to functions of finite type gives the transcendental version of Proposition 1.1.

More precisely let $\widehat{\mathcal{B}} \subset \mathcal{B}$ be the class of entire transcendental functions defined in [RRRS11] for which the escaping set consists of curves, known as dynamic rays (see Section 2). Class $\widehat{\mathcal{B}}$ contains all functions in $\mathcal{B}$ which are either of finite order or composition of functions of finite order in class $\mathcal{B}$. A dynamic ray $G$ is periodic if $f^{n}(G)=G$ for some $n \in \mathbb{N}$ and we say that a ray lands if $\bar{G} \backslash G=\left\{z_{0}\right\} \subset \mathbb{C}$. Finally a rationally invisible repelling point is a repelling periodic point which is not the landing point of any periodic ray (see Remark 3.4.

Proposition 1.2 (Fatou-Shishikura inequality). Let $f$ be an entire transcendental map in class $\widehat{\mathcal{B}}$ with $N<\infty$ singular values, whose periodic rays land. Then $f$ has at most $N$ non-repelling cycles.

Moreover for each non-repelling cycle $\mathcal{X}$ there exists at least one singular orbit $\left\{f^{n}(s)\right\}_{n \in \mathbb{N}}$ with $s \in S(f)$ which is associated to $\mathcal{X}$ in the sense that

(1) $\left\{f^{n}(s)\right\}_{n \in \mathbb{N}}$ accumulates on every element of $\mathcal{X}$ (or, the boundaries of the Siegel disks containing $\mathcal{X}$ );

(2) $\left\{f^{n}(s)\right\}_{n \in \mathbb{N}}$ does not accumulate on any other non-repelling cycle, nor on any rationally invisible repelling point, nor on any point on the boundary of a Siegel disk $\Delta \notin \mathcal{X}$ (provided the point is not on a periodic ray or a periodic point).

Let us make some comments on the statement above. For functions of finite type, Proposition 1.2 gives more information than the classical Fatou-Shishikura inequality in [EL92], since it associates individual singular orbits to individual non-repelling cycles rather than relying on a global counting of the number of singular values and the number of non-repelling cycles. However our proof requires the existence of dynamic rays (which is ensured by assuming that $f$ is a composition of functions of finite order in class $\mathcal{B}$ ), and that periodic dynamic rays land. Although it is expected that all periodic rays land except those whose forward orbit hits a singular value (as it is the case for polynomials), so far this has only been proven for the family $e^{z}+c$ Rem06]. The hypothesis of landing of periodic rays is implied by the requirement that the postsingular set 


$$
\mathcal{P}(f):=\overline{\bigcup_{n \in \mathbb{N}, s \in S(f)} f^{n}(s)}
$$

is bounded, but the latter is in general a much stronger hypothesis. For example, when the map is $f(z)=e^{z}+c$, having bounded postsingular set implies non-recurrence of the asymptotic value.

Observe also that the statement is most meaningful when thinking of the interplay between singular orbits and irrationally indifferent cycles. Indeed, if $\mathcal{X}$ is an attracting or parabolic cycle, the cycle of its attracting basins contains a singular value [Mil06, so to each attracting or parabolic cycle is associated trivially a singular orbit in the sense Proposition 1.2. Conversely, it is obvious that the singular orbits accumulating on a Cremer cycle or a cycle of boundaries of Siegel disks cannot intersect any attracting or parabolic basin.

Finally we remark that it is not known whether it is possible for boundaries of Siegel disks to contain periodic points or points belonging to periodic rays. If this were never the case, the special case at the end of (2) could be removed.

Since our methods are dynamical and do not rely on perturbations in finite-dimensional parameter spaces, we also obtain results for functions with infinitely many singular values. In order to be able to state such results we need some additional understanding of the structure of the dynamical plane for a function $f \in \widehat{\mathcal{B}}$ whose periodic rays land.

We say that a periodic dynamic ray $G$ (of period $p$ ) lands alone if its landing point is not the landing point of any other dynamic ray (of period $p$ ). By recent results in [BRG17, the concept of landing alone is independent of the period, so we will omit it. For any $p \geq 1$, consider the closed graph $\Gamma_{p}$ formed by rays which are fixed under $f^{p}$ and which do not land alone, together with their landing points. The graph $\Gamma_{p}$ disconnects $\mathbb{C}$ into open unbounded regions, called basic regions (for $f^{p}$ ).

The Separation Theorem in BF15] (see Section 2, and [GM93 for polynomials), states that, even though the number of fixed rays by $f^{p}$ is infinite, the number of basic regions is finite, and each of them contains exactly one of the following: either a parabolic basin invariant under $f^{p}$; or an attracting point fixed by $f^{p}$; or a Siegel point fixed by $f^{p}$; or a Cremer point fixed by $f^{p}$; or a repelling point fixed by $f^{p}$ which is not the landing point of any fixed ray $\left(\right.$ of $f^{p}$ ). Following [GM93, the attracting, Siegel, Cremer or repelling fixed point is called an interior fixed point (for $f^{p}$ ), and the invariant parabolic basin is called a virtual fixed point (for $f^{p}$ ).

Our main theorem is the following:

Theorem 1.3 (Singular orbits trapped in basic regions). Let $f$ be an entire transcendental map in class $\widehat{\mathcal{B}}$ whose periodic rays land. Let $\mathcal{X}$ be a cycle of Siegel disks, attracting basins, parabolic basins or Cremer points of period $q$ and let $p$ be any multiple of $q$. Let $\left\{B_{i}\right\}_{i=0 \ldots q-1}$ be the basic regions for $f^{p}$ containing the elements of $\mathcal{X}$. Then, up to relabeling the indices, at least one of the following is true.

(1) There exists a singular value $v$ for $f$ such that $v \in \bigcup_{i=0}^{q-1} B_{i}$, say $v \in B_{0}$, and such that $f^{n}(v) \in B_{i}$ whenever $n \bmod q=i$. The orbit of $v$ accumulates either on the non-repelling cycle or on the boundary of the cycle of Siegel disks. 
(2) There are infinitely many singular values $s_{j}$ for $f$ in at least one of the basic regions $B_{i}$, say $B_{0}$, and a sequence $n_{j} \underset{j \rightarrow \infty}{\longrightarrow} \infty$ such that $f^{n}\left(s_{j}\right) \in B_{i}$ whenever $n \bmod q=i$ for all $n \leq n_{j}$. The orbits $\left\{f^{n}\left(s_{j}\right)\right\}_{j \in \mathbb{N}, n \leq n_{j}}$ accumulate either on the non-repelling interior cycle, or on the boundary of the associated Siegel disk.

The first case always occurs if $\mathcal{X}$ is attracting or parabolic or if $f$ has only finitely many singular values.

Moreover, in case (1), the orbit of $v$ does not accumulate on any other interior periodic cycle or on any point on the boundary of a Siegel disk $\Delta \notin \mathcal{X}$ (provided the point is not on a periodic ray or a periodic point).

Theorem 1.3 implies for example that if $f$ has infinitely many singular values, all but finitely many of which are in an attracting or parabolic basins, then $f$ has at most as many additional non-repelling cycles as the number of 'free' singular values. This fact is certainly not surprising but we believe it does not follow directly from the results by Eremenko and Lyubich [EL92], whose proof uses perturbation in a finite-dimensional parameter space.

If all singular values but finitely many are escaping, the situation is not clear. For example, let $\Delta$ be a bounded Siegel disk and let $\mathcal{C}_{n}$ be a sequence of finite coverings of $\partial \Delta$ by balls of radius $1 / n \rightarrow 0$. Then if we have infinitely many escaping singular values $\left\{s_{j}\right\}_{j \in \mathbb{N}}$ we can make the singular orbit of $s_{j}$ visit all balls in $\mathcal{C}_{j}$ before escaping infinity.

We believe that our methods work also when $f \in \mathcal{B} \backslash \widehat{\mathcal{B}}$. In that case the role of dynamic rays is taken by analogous, non-pathconnected objects called dreadlocks [BRG17]. One would need to prove the separation theorem using dreadlocks instead of rays and assume that periodic dreadlocks land. Such extension would remove the function theoretical assumption on $f$.

The paper is organized as follows. Section 2 contains some of the background results that will be used throughout the paper. Section 3 is aimed at proving the main result (Theorem 1.3 and some corollaries including Proposition 1.2

\section{Acknowledgements}

We wish to thank Lasse Rempe-Gillen and Mitsuhiro Shishikura for helpful discussions. We are grateful to the Institut de Matemàtica de la Universitat de Barcelona (IMUB) for its hospitality.

\section{Background}

Let $f$ be an entire transcendental function in class $\mathcal{B}$ and let $D$ be a disk containing $S(f)$. The connected components of $f^{-1}(\mathbb{C} \backslash \bar{D})$ are called tracts and they are unbounded and simply connected. By definition for any tract $T$ we have that $f: T \rightarrow \mathbb{C} \backslash \bar{D}$ is an unbranched covering of infinite degree. Let $\mathcal{T}$ denote the union of all tracts. One can easily show that there exists a (piecewise analytic) curve $\delta \subset \mathbb{C} \backslash(\overline{D \cup \mathcal{T}})$ connecting $\delta$ to $\infty$ Rot05. The preimages of $\mathbb{C} \backslash(\bar{D} \cup \delta)$ are called fundamental domains and, since fibers are discrete, it follows that only 
finitely many tracts and fundamental domains intersect $D$. For any fundamental domain $F$ we have that

$$
f: F \rightarrow \mathbb{C} \backslash(\bar{D} \cup \delta)
$$

is a biholomorphism. We refer to [EL92], [RRRS11] and [BF15] for details and other properties.

Functions in class $\mathcal{B}$ have expansive properties near infinity inside fundamental domains, as shown in the following lemma.

Lemma 2.1 ([BF15, Proposition 2.6]). Let $\mathcal{F}=\left\{F_{\alpha}\right\}_{\alpha=1 \ldots N}$ be a finite collection of fundamental domains. Then for any $R$ large enough there exists an (analytic) Jordan curve $\gamma$ in $\{|z|>R\}$ such that the preimages $\gamma_{\alpha} \subset F_{\alpha}$ of $\gamma$ are contained in the bounded connected component of $\mathbb{C} \backslash \gamma$.

Fundamental domains are endowed with a natural cyclic order, and can be used to define symbolic dynamics on the set of escaping points (or at least, those which stay far enough from $D$ if the postsingular set is not bounded). This allows to define sets of escaping points which share the same itinerary. If $f \in \widehat{\mathcal{B}}$, it is shown in [RRRS11] that the tracts have a nice enough geometry to prove that these sets of escaping points are injective curves, called dynamic rays, and that each escaping point belongs to a dynamic ray or a preimage thereof. More precisely, let $\Sigma$ be the set of infinite sequences whose symbols are the fundamental domains of $f$, and let $\sigma$ be the left-sided shift map acting on $\Sigma$. The elements of $\Sigma$ are called $a$ ddresses. An address is bounded if it takes values over a finite family of fundamental domains, and periodic if it is a periodic sequence. The following statement summarizes the relation between these objects.

Theorem 2.2 ([RRRS1] $)$. Let $\widehat{\mathcal{B}} \subset \mathcal{B}$ be the class of maps formed by finite compositions of finite order maps in $\mathcal{B}$. Then there exists $\mathcal{N} \subset \Sigma$ such that the if $z \in I(f)$, then for $n$ large enough $f^{n}(z)$ belongs to an injective unbounded curve $G_{\underline{s}} \subset I(f)$ called the dynamic ray of address $\underline{s}$ for some $\underline{s} \in \mathcal{N}$. The correspondence $\underline{s} \mapsto G_{\underline{s}}$ is injective and $G_{\underline{s}} \cap G_{\tilde{s}}=\emptyset$ for $\underline{s} \neq \tilde{s}$. Dynamic rays satisfy the relation

$$
f\left(G_{\underline{s}}\right)=G_{\sigma \underline{s}} .
$$

A dynamic ray $G_{\underline{s}}$ is periodic if $\underline{s}$ is periodic, and has bounded address if $\underline{s}$ is bounded. The set $\mathcal{N}$ of addresses which are realized depends on the class of $f$ as defined in [EL92, Rem09], but $\mathcal{N}$ always contains the set of bounded addresses [Rem08, BK07] and addresses which are exponentially bounded in the sense of [BRG17]. For the exponential family $\mathcal{N}$ is completely characterized [SZ03]. For more on the characterization of $\mathcal{N}$ see [ABR17].

The initial idea of finding curves in the escaping set goes back to DT86, DK84, BK07. For functions with not as beautiful a geometry as functions in class $\widehat{\mathcal{B}}$, the role of dynamic rays is played by more general connected sets of escaping points called dreadlocks [BRG17.

We say that an unbounded set $X$ is asymptotically contained in another unbounded set $U$ if and only if there exists $R$ such that $X \cap\{z \in \mathbb{C}:|z|>R\} \subset U$. It is not hard to see that a ray $G_{\underline{s}}$ of address $\underline{s}=F_{0} F_{1} \ldots$ is asymptotically contained in the fundamental domain $F_{0}$. Also, for each fundamental domain $F$ there exists a unique fixed ray with address $\bar{F}$ (see [Rem08, [BK07] and [BF15, Lemma 2.3]) and which is asymptotically contained in $F$. 
The proof of Theorem 1.3 uses strongly the following theorem.

Theorem 2.3 (Separation Theorem [BF15]). Let $p \geq 1$ and $f \in \widehat{\mathcal{B}}$ and assume that all fixed rays of $f^{p}$ land. Then there are finitely many basic regions for $f^{p}$, and each basic region contains exactly one interior fixed point or virtual fixed point of $f^{p}$.

Theorem 2.3 was inspired by an analogous Separation Theorem by Goldberg and Milnor GM93 for polynomials with connected Julia sets, a condition equivalent to requiring that the postcritical set is bounded and which implies that all periodic rays land. So in the transcendental setting, the assumption that all periodic rays land is weaker than than the hypothesis that the postsingular set is bounded.

Goldberg-Milnor's Separation Theorem and Theorem 2.3 have many corollaries, including that parabolic points are always landing points of periodic dynamic rays, and that hidden components of a Siegel disk are preperiodic to the Siegel disk itself (see [CR16] and [BF17] for an application of this fact to the existence of critical points on the boundary of Siegel disks). See also [Kiw00].

\section{Singular Values and Basic Regions}

In this section we prove Theorem 1.3 and Proposition 1.2. Consider an entire transcendental map $f \in \tilde{\mathcal{B}}$ whose periodic rays land, and consider the basic regions for $f^{p}$ for some $p \in \mathbb{N}$. By the Separation Theorem 2.3, each basic region contains exactly one interior fixed point for $f^{p}$ or an attracting parabolic basin fixed under $f^{p}$.

We shall make a further distinction between different types of basic regions. See Figure 1.

Definition 3.1 (Basic regions of transcendental and polynomial type). A basic region $B$ is called of polynomial type if $B \cap(\mathbb{C} \backslash \mathcal{T})$ is bounded and of transcendental type otherwise.

The following proposition is not surprising, and we will not need it in the sequel, but we include it because it illustrates the fact that transcendental behaviour, as for example the presence of unbounded Fatou components, only appears associated to basic regions of transcendental type.

Proposition 3.2 (Bounded Fatou Components). Let $Q$ be a periodic Siegel disk, a periodic attracting basin, or a periodic parabolic basin of period $q$ which is contained in a basic region $B$ of polynomial type for $f^{p}$, with $p$ multiple of $q$. Then $Q$ is bounded, and either $\partial Q \cap \partial B$ is empty or it is contained in the set of boundary fixed points.

Proof. Without loss of generality we can assume that the period is 1 and that $B$ is a basic region for $f$. Let $z_{0}$ be the attracting, indifferent or parabolic interior fixed point for $B$. We will only prove the case in which $z_{0}$ is the center of a Siegel Disk $\Delta$, since the other two cases are very similar.

Since $B$ is of polynomial type and rays are asymptotically contained in fundamental domains, $B$ intersect finitely many fundamental domains. Indeed, the boundary of $B$ is 


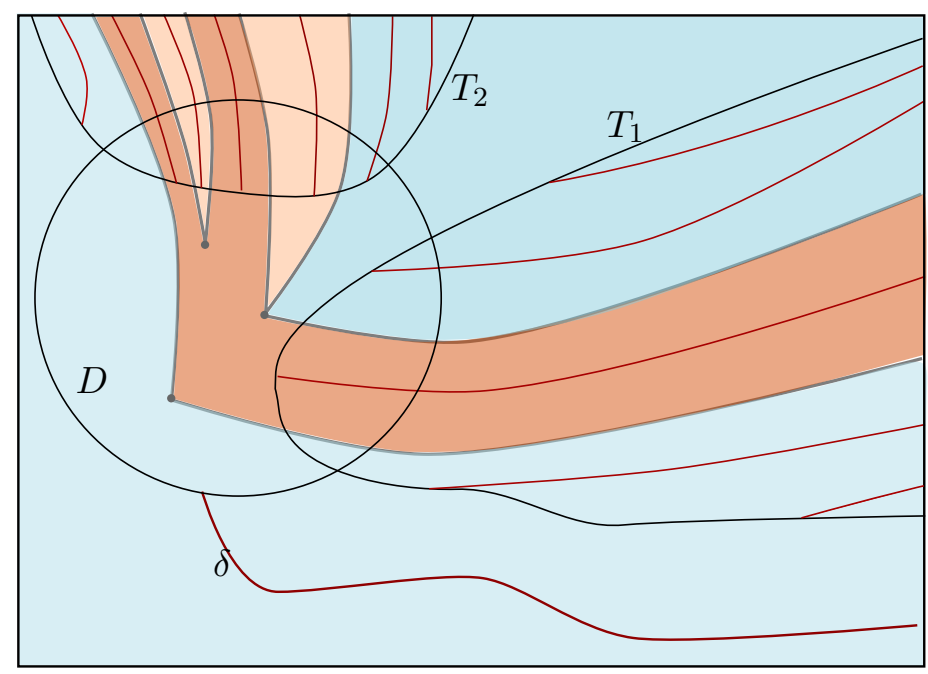

Figure 1: Basic regions of polynomial type (orange) and transcendental type (blue). Fixed rays and their landing points are shown in grey. In red, the curve $\delta$ and its preimages, which, together with the track boundaries (black) bound the fundamental domains.

made of finitely many rays pairs union the point $\infty$. While moving along the boundary of $B$ counterclockwise (passing trough infinity when moving from one ray pair to the other), any two consecutive rays which do not land together belong to the same tract (otherwise $B \backslash \mathcal{T}$ would be unbounded), hence there are only finitely many fundamental domains between them. Since $\partial B$ contains finitely many ray pairs and fundamental domains have a cyclic order, $B$ intersect finitely many fundamental domains.

Let $\gamma, \gamma_{\alpha}$ be as in Lemma 2.1. Consider the region $\widehat{B}$ obtained by cutting $B$ with the arcs $\gamma_{\alpha}$. By choice of $\gamma_{\alpha}, \widehat{B}$ is contained in the bounded connected component of $\mathbb{C} \backslash \gamma$, hence $f\left(\gamma_{\alpha}\right) \cap \widehat{B}=\emptyset$. Let us start by showing that $\Delta \subset \widehat{B}$.

Let $\phi: \mathbb{D} \rightarrow \Delta$ be the Riemann map conjugating $f$ to a rotation, and for all $r<1$ let $V_{r}:=\phi\left(\mathbb{D}_{r}\right)$. Let $r_{0}:=\sup \left\{r: V_{r} \subset \widehat{B}\right\}$. Observe that $\Delta \subset \widehat{B}$ if and only if $r_{0}=1$. Suppose that $r_{0}<1$. Since $\Delta$ is fully contained in $B$, we would have that $\partial \phi\left(V_{r_{0}}\right) \cap \gamma_{\alpha} \neq \emptyset$. But since $\overline{V_{r_{0}}} \subset \overline{\widehat{B}}$ is forward invariant, we have that $f\left(\overline{V_{r_{0}}}\right) \subset \overline{\widehat{B}}$, while $f\left(\gamma_{\alpha}\right) \subset \mathbb{C} \backslash \widehat{\widehat{B}}$ by choice of $\gamma_{\alpha}$, this gives a contradiction. So $r_{0}=1, \Delta$ is fully contained in $\widehat{B}$ and in particular it is bounded. Since it is bounded its boundary cannot contain escaping points, and since it is contained in $\widehat{B}$ and forward invariant it cannot intersect $r_{\alpha}$ (again because $f\left(r_{\alpha}\right) \subset \mathbb{C} \backslash \widehat{B}$ ). So $\partial \Delta \cap \partial B$ contains at most the boundary fixed points.

We observe that one could prove Proposition 3.2 also by using weakly polynomial-like maps.

Remark 3.3. It seems to be a difficult problem to know whether there can be (repelling) periodic points on the boundaries of Siegel disks. For polynomials, it is known that if such a case occurs, then the boundary of the Siegel Disk is an indecomposable continuum. By Pérez-Marco [PM97], there are no periodic points on the boundary of a Siegel disk if the latter has a neighborhood on which $f$ and $f$ inverse are well defined and univalent (he calls 
this Siegel Disks of type I). It is also not unlikely to think that the boundary of unbounded Siegel Disk could contain escaping points. Observe that rays can not only intersect, but even be contained in the boundaries of attracting basins; for example, when the map $\lambda e^{z}$ has an attracting fixed point with a completely invariant basin of attraction, it is known that the Julia set equals the boundary of such basin and consists of curves of escaping points together with their landing points. Proposition 3.2 ensures that there cannot be escaping points on the boundary of Fatou components which are in a basic region of polynomial type.

We now proceed to prove the main result in the paper.

Proof of Theorem 1.3. Let $\mathcal{X}$ be as in the statement. If it is a cycle of attracting or parabolic basins there is nothing to prove since each such cycle contains a singular orbit. The proof is a refinement of the classical fact that Cremer points and the boundaries of Siegel disks are contained in the postsingular set (see Corollary 14.4 in [Mil06, Corollary 14.4] for Siegel Disks and [Bea91, Theorem 9.3.4] for Cremer points).

Siegel case. Let $\mathcal{X}=\Delta_{0}, \Delta_{1}, \ldots \Delta_{q-1}$ be a cycle of Siegel disks of period $q$. Fix any $p$ multiple of $q$ and let $\left\{B_{i}\right\}_{i=0 \ldots q}$ be the $q$ basic regions for $f^{p}$ containing the images $\Delta_{1} \ldots \Delta_{q-1}$ of $\Delta=\Delta_{0}$, with $f\left(\Delta_{i}\right)=\Delta_{i+1}$ and all indices are taken modulo $q$. Let us define the set of singular values $S_{B}:=S(f) \cap\left(\cup B_{i}\right)$, and let us define

$$
\mathcal{P}_{B}:=\overline{\bigcup_{s \in \mathcal{S}_{B}, n \leq n_{s}} f^{n}(s)}
$$

where $n_{s} \leq \infty$ is the largest integer such that $f_{s}^{n}(s) \in \cup B_{i}$ for all $n \leq n_{s}$.

Let $w \in \partial \Delta_{0} \backslash \partial B_{0}$ : such a point exists, or otherwise we would have $\Delta_{0}=B_{0}$, which is impossible ( $B_{0}$ contains, for example, rays, which cannot intersect $\left.\Delta_{0}\right)$.

Let us first show that $w \in \mathcal{P}_{B}$. Suppose by contradiction that there exists a simply connected neighborhood $U$ of $w$ with $U \cap \mathcal{P}_{B}=\emptyset$. Let us see that this implies that for each $n$ there is a unique univalent inverse branch $\phi_{n}$ of $f^{-n}$ such that $\phi_{n}\left(\Delta_{0} \cap U\right) \subset \Delta_{-n} \bmod q$; this would be the same as in Mil06 if we were considering $\mathcal{P}$ instead of $\mathcal{P}_{B}$, but in our case requires a further argument. Since $w \in \partial \Delta_{0} \backslash \partial B_{0}$, up to restricting $U$ we can assume that $U \subset B_{0}$. Observe that any preimage $V$ of $U$ under $f^{n}$ with $V \cap \Delta_{i} \neq \emptyset$ for some $i$, is fully contained in $B_{i}$. (Indeed, the graph $\Gamma_{p}$ formed by the closure of the rays invariant under $f^{p}$ is invariant under $f$. So, if there was a point $z \in \partial\left(\cup B_{i}\right) \cap V \subset\left(\Gamma_{p} \cap V\right)$, by forward invariance of $\Gamma_{p}$ we would have $f(z) \in U \cap \Gamma_{P}$, a contradiction.)

So for each $n$ there is a unique univalent inverse branch $\phi_{n}$ of $f^{-n}$ such that $\phi_{n}(\Delta \cap U) \subset$ $\Delta_{-n} \bmod q$; the $\left\{\phi_{n}\right\}$ form a normal family by Montel Theorem because they can be chosen to omit two values (say, a repelling orbit of period at least two which does not intersect $U$ ). Hence a subsequence $\phi_{n_{k}}$ converges locally uniformly to an analytic limit map $\phi: U \rightarrow \phi(U)$.

The map $\phi$ is not constant because no subsequence of $\phi_{n}$ is converging to a constant on $\Delta_{0}$. On the other hand, let $D \Subset \phi(U)$ be a slightly smaller topological disk still intersecting the Julia set. Then for $n$ large, $\phi_{n}(U) \supset D$, hence $\left.f^{n}\right|_{D} \subset U$ for infinitely many $n$, contradicting the fact that $D$ intersects $J(f)$.

So $\phi_{n}$ is defined and univalent on $U$ only for $n \leq n_{1}$ with $n_{1}$ maximal (possibly, $n_{1}=0$ if $U$ contains singular values). Since univalent branches in a simply connected open set $V$ are 
well defined and univalent only if $V$ does not contain singular values, it follows that there is a singular value $s_{1}$ for $f$ such that $s_{1} \in \phi_{n_{1}}(U)$. In particular, $f^{n}\left(s_{1}\right) \in f^{n}\left(\phi_{n_{1}}(U)\right) \subset \cup B_{i}$ for all $n \leq n_{1}$. The same argument can be repeated for a decreasing sequence $\left\{U_{j}\right\}$ of nested simply connected neighborhoods of $w$, obtaining an infinite sequence of points $w_{j}=f^{n_{j}}\left(s_{j}\right)$ accumulating on $w$ with $s_{j} \in \mathcal{S}_{B}$ and $f^{n}\left(s_{j}\right) \in \cup B_{i}$ for $n \leq n_{j}$.

Now there are two (non-exclusive) cases. Suppose first that $s_{j}=s$ for some $s \in S(f)$ and infinitely many $j$. In this case $n_{j} \rightarrow \infty, f^{n}(s) \in \cup B_{i}$ for all $n$ and case (1) occurs. If this case does not occur, then there are infinitely many distinct $s_{j} \in S_{B}$ such that $f^{n}\left(s_{j}\right) \in \cup B_{i}$ for $n \leq n_{j}$ and we are in case (2). In this case either $n_{j} \rightarrow \infty$ or, if $n_{j}$ has a bounded subsequence, case (1) occurs. Indeed, if $n_{j}$ has a bounded subsequence, there is some minimal $N>0$ and a subsequence $j_{k}$ such that $f^{N}\left(s_{j_{k}}\right)$ converges to $w$. This implies that $s_{j_{k}} \rightarrow \phi_{N}(w)$ hence since $\mathcal{S}(f)$ is closed, $\phi_{N}(w) \in \mathcal{S}(f) \cap \partial \Delta$. Since the union of the boundaries of the Siegel disks in the forward orbit of $\Delta$ is forward invariant, $\phi_{N}(w)$ satisfies the hypothesis of case (1).

Since singular values of $f^{q}$ are the first $q-1$ images of singular values for $f$, it is directly implied by the construction that each $B_{i}$ contains a singular value $s_{i}$ for $f^{q}$ for which $f^{n q}\left(s_{i}\right) \in$ $B_{i}$ for all $n \in \mathbb{N}$ such that $n q \leq n\left(s_{i}\right)$.

Cremer case. Let $z_{0}$ be a Cremer fixed point. The proof is the same as in the Siegel case except that the inverse branches $\phi_{n}$ are defined so as to fix $z_{0}$, and the limit function $\phi$ is non-constant because $\left|\phi_{n}^{\prime}\left(z_{0}\right)\right|=1$ for all $n$.

Now suppose we are in case (1) and let $v \in B_{0}$ be the singular value for the cycle $\mathcal{X}$. Let $\mathcal{Y}$ be any other interior cycle of period $\ell$. Let $p=\ell \cdot q$. By the Separation Theorem, elements of $\mathcal{X}$ and elements of $\mathcal{Y}$ belong to different basic regions for $f^{p}$ and therefore the orbit of $v$ is disjoint from the basic regions containing $\mathcal{Y}$.

Remark 3.4. A repelling periodic point which is not the landing point of any periodic ray of any period is called rationally invisible and is an interior fixed point for $f^{p}$ for all $p$. It is expected that every repelling periodic orbit is the landing point of at least one but at most finitely many periodic rays of the same period, although partial results are only available when the postsingular set is bounded [Hub93], [BL14], [BRG17]). Theorem 1.3 together with the Separation Theorem implies that the singular orbits given by case (1) cannot accumulate on any rationally invisible repelling periodic point, since the latter belongs to different basic regions for $f^{p}$ than $\mathcal{X}$ when $p$ is large enough.

The next Proposition is another practical application of the philosophy that polynomialtype regions are associated to polynomial-type behaviour.

Proposition 3.5 (Regions of polynomial type). Under the assumptions of Theorem 1.3 , the first case always occurs if at least one of the $B_{i}$ is a basic region of polynomial-type. In this case the singular value given by Theorem 1.3 is in fact a critical value.

Proof. Suppose that $B_{0}$ is a basic region of polynomial type. Consider the cut region $\widehat{B}_{0} \subset B_{0}$ as in Proposition 3.2. Observe that $\partial \widehat{B}_{0} \backslash \partial B_{0}=\left\{\gamma_{\alpha}\right\}$. In the construction above notice that $\phi_{n}(U) \subset \widehat{B}_{0}$ for all $n$, otherwise $\phi_{n}(U)$ would contain points in $\gamma_{\alpha}$ for some minimal $n$ which is impossible because $f\left(\gamma_{\alpha}\right) \cap \widehat{B}_{0}=\emptyset$ and $\phi_{n-1}(U) \subset \widehat{B}_{0}$. This implies that all singular values 
preventing the continuation of the corresponding inverse branches are critical values, since finite non-critical preimages of singular values do not obstruct the extension. Since $\widehat{B}_{0}$ is bounded it contains only finitely many critical points, hence finitely many singular values, and one of them has to accumulate on $\partial \Delta$ infinitely many times implying the occurrence of case (1).

From the proof of Theorem 1.3 we obtain the following corollary, which says that every basic region $B$ for $f^{p}$ whose interior fixed point is non-repelling contains either infinitely many singular values for $f^{p}$, or at least a singular value for $f^{p}$ which returns to $B$ infinitely many times.

Corollary 3.6 (Singular Values and basic regions). Let $f$ be a polynomial or an entire transcendental map in class $\widehat{\mathcal{B}}$ whose periodic rays land. Let $B$ be a basic region for $f^{p}$ whose interior fixed point $z_{0}$ is non-repelling, or which contains an attracting parabolic basin. Then at least one of the two following cases occur:

1. $B$ contains at least one singular value $s$ for $f^{p}$ whose orbit $f^{n p}(s)$ is contained in $B$ for all $n \geq 0$ and accumulates either on the parabolic, attracting or Cremer fixed point, or on the boundary of the associated Siegel disk.

2. B contains infinitely many singular values $s_{j}$ for $f^{p}$ such that $f^{p n}\left(s_{j}\right) \in B$ for all $n \leq n_{j} \rightarrow \infty$ as $j \rightarrow \infty$, and $\left\{f^{p n_{j}}\left(s_{j}\right)\right\}_{n, j}$ accumulates either on the interior periodic point, or on the boundary of the associated Siegel disk.

The first case always occurs if $z_{0}$ is attracting, or $B$ contains a parabolic basins, or $B$ is a basic region of polynomial-type, or $f$ has finitely many singular values.

Proof of Corollary 3.6. Let $B$ be a basic region for $f^{p}$ whose interior fixed point is nonrepelling. If the interior fixed point is attracting, or if $B$ contains a parabolic basin invariant under $f^{p}$, there is nothing to prove, since in this case the cycle of basins contains a singular orbit for $f$. So we can assume that $B$ contains either a Cremer point $z_{0}$ or a Siegel disk $\Delta$ of period $q$, which necessarily divides $p$ since it is fixed under $f^{p}$. The claim then follows from Theorem 1.3, and the fact that $S\left(f^{p}\right)=S(f) \cup f(S(f)) \cup \ldots \cup f^{p}(S(f))$.

The fact that polynomial type basic regions are always in case (1) follows from Proposition 3.5 .

As the final corollary of Theorem 1.3 we obtain the improvement on the classical Fatou Shishikura mentioned in the introduction.

Proof of Proposition 1.2. Suppose by contradiction that $f$ has $q$ singular values and more than $q$ non-repelling cycles (possibly infinitely many). Take $N+1$ of them and let $p$ be the product of their periods. Each element in each of the $N+1$ cycles is fixed by $f^{p}$ hence belongs to a different basic region for $f^{p}$. In particular, there are $N+1$ disjoint collections of basic regions which by Theorem 1.3 have to contain each a singular value for $f$ as well as its entire orbit, giving a contradiction. 
Let $\mathcal{X}$ be any non-repelling cycle of period $q$ and let $s$ be the singular value given by Theorem 1.3. Let $\mathcal{Y}$ be any other non-repelling cycle or a rationally invisible repelling periodic cycle of period $\ell$. Let $p=\ell \cdot q$. Then elements of $\mathcal{X}$ and elements of $\mathcal{Y}$ belong to different basic regions for $f^{p}$. By part 1. in Theorem 1.3 the orbit of $s$ is disjoint from the basic regions containing $\mathcal{Y}$.

\section{References}

[ABR17] Simon Albrecht, Anna M. Benini, and Lasse Rempe-Gillen, Conjugacy classes of finite-order transcendental entire functions, in preparation, 2017.

$\left[\mathrm{BCL}^{+} 16\right]$ Alexander Blokh, Doug Childers, Genadi Levin, Lex Oversteegen, and Dierk Schleicher, An extended fatou-shishikura inequality and wandering branch continua for polynomials, Adv. Math. 288 (2016), 1121-1174.

[Bea91] Alan F. Beardon, Iteration of rational functions, Graduate Texts in Mathematics, vol. 132, Springer-Verlag, New York, 1991, Complex analytic dynamical systems. MR 1128089

[BF15] Anna Miriam Benini and Núria Fagella, A separation theorem for entire transcendental maps, Proc. Lond. Math. Soc. (3) 110 (2015), no. 2, 291-324.

[BF17] Anna Miriam Benini and Nuria Fagella, Singular values and bounded siegel disks, Mathematical Proceedings of the Cambridge Philosophical Society, Cambridge University Press, 2017, pp. 1-17.

[BK07] Krzysztof Barański and Bogusława Karpińska, Coding trees and boundaries of attracting basins for some entire maps, Nonlinearity 20 (2007), no. 2, 391-415.

[BL14] Anna Miriam Benini and Mikhail Lyubich, Repelling periodic points and landing of rays for post-singularly bounded exponential maps, Annales de l'Institut Fourier 64 (2014), no. 4, 1493-1520.

[BRG17] Anna Miriam Benini and Lasse Rempe-Gillen, A landing theorem for entire functions with bounded post-singular set, preprint, arXiv:1711.10780 [math.DS] (2017).

[CR16] Arnaud Chéritat and Pascale Roesch, Herman's condition and critical points on the boundary of siegel disks of bicritical polynomials, Comm. Math. Phys. 344 (2016), no. 2, 397-426.

[DH85] Adrien Douady and John Hamal Hubbard, On the dynamics of polynomial-like mappings, Annales scientifiques de l'Ecole normale supérieure, vol. 18, Elsevier, 1985, pp. 287-343.

[DK84] Robert L. Devaney and Michał Krych, Dynamics of $\exp (z)$, Ergodic Theory Dynam. Systems 4 (1984), no. 1, 35-52.

[DT86] Robert L. Devaney and Folkert Tangerman, Dynamics of entire functions near the essential singularity, Ergodic Theory Dynam. Systems 6 (1986), no. 4, 489-503. 
[EL92] Alexandre È. Eremenko and Mikhail Yu. Lyubich, Dynamical properties of some classes of entire functions, Ann. Inst. Fourier (Grenoble) 42 (1992), no. 4, 9891020.

[Eps99] Adam Epstein, Infinitesimal thurston rigidity and the fatou-shishikura inequality, arXiv preprint math/9902158 (1999).

[Fat20] P. Fatou, Sur les équations fonctionnelles, Bull. Soc. Math. France 48 (1920), 208-314. MR 1504797

[GK86] Lisa R. Goldberg and Linda Keen, A finiteness theorem for a dynamical class of entire functions, Ergodic Theory Dynam. Systems 6 (1986), no. 2, 183-192.

[GM93] Lisa R. Goldberg and John Milnor, Fixed points of polynomial maps. II. Fixed point portraits, Ann. Sci. École Norm. Sup. (4) 26 (1993), no. 1, 51-98.

[Hub93] John Hamal Hubbard, Local connectivity of Julia sets and bifurcation loci: three theorems of J.-C. Yoccoz, Topological methods in modern mathematics (Stony Brook, NY, 1991), Publish or Perish, Houston, TX, 1993, pp. 467-511.

[Kiw00] Jan Kiwi, Non-accessible critical points of cremer polynomials, Ergodic Theory and Dynamical Systems 20 (2000), no. 5, 1391-1403.

[Mil06] John Milnor, Dynamics in one complex variable, third edition ed., vol. 160, Annals of Mathematics Studies, Princeton University Press, Princeton, NJ, 2006.

[PM97] Ricardo Pérez-Marco, Fixed points and circle maps, Acta Math. 179 (1997), no. 2, 243-294.

[Rem06] Lasse Rempe, A landing theorem for periodic rays of exponential maps, Proc. Amer. Math. Soc 134 (2006), no. 9, 2639-2648.

[Rem08] Lasse Rempe, Siegel disks and periodic rays of entire functions, J. Reine Angew. Math. 624 (2008), 81-102.

[Rem09]__ Rigidity of escaping dynamics for transcendental entire functions, Acta Math. 203 (2009), no. 2, 235-267.

[Rot05] Günter Rottenfuer, Dynamical Fine Structure of entire transcendental functions, doctoral thesis, International University Bremen, 2005.

[RRRS11] Günter Rottenfuer, Johannes Rückert, Lasse Rempe, and Dierk Schleicher, Dynamic rays of bounded-type entire functions, Ann. of Math. (2) 173 (2011), no. 1, $77-125$.

[Shi87] Mitsuhiro Shishikura, On the quasiconformal surgery of rational functions, Annales scientifiques de l'École Normale Supérieure, vol. 20, Elsevier, 1987, pp. 1-29.

[SZ03] Dierk Schleicher and Johannes Zimmer, Escaping points of exponential maps, J. London Math. Soc. (2) 67 (2003), no. 2, 380-400. 